\title{
Nanosilver against fungi. Silver nanoparticles as an effective biocidal factor*
}

\author{
Jolanta Pulit ${ }^{\boxplus}$, Marcin Banach¹, Renata Szczygłowska² and Mirosław Bryk ${ }^{3}$ \\ ${ }^{1}$ Cracow University of Technology, Institute of Inorganic Chemistry and Technology, Kraków, Poland, 2Silesian Environmental Ph.D. Studies Cen- \\ tre, Central Mining Institute, Katowice, Poland, ${ }^{3}$ The Implementing Company Kraków, Poland
}

The work presents a method of obtaining an aqueous raspberry extract as well as its physicochemical and analytical characteristics. The paper also contains a description of the method of preparation of nanosilver suspensions based on this extract. The raspberry extract served as a source of phenolic compounds which acted as both reducing and stabilizing agents. Suspensions of silver nanoparticles were obtained with the use of chemical reduction method. The silver ions concentration, $\mathrm{pH}$ value and temperature of samples incubation were independent variables. The next step of the research was to measure the antifungal activity of the received silver nanoparticles as well as to perform a mycological efficacy resistance analysis of the tested preparations in relation to different concentrations of nanostructured silver. Tests were conducted in compliance with the Eucast guidelines. The results of microbiological study of (the samples') biocidal effect against Cladosporium cladosporoides and Aspergillus niger are described. It was found that using nanosilver suspension at the concentration of $50 \mathrm{ppm}$ inhibited the growth of Cladosporium cladosporoides and Aspergillus niger by $90 \%$ and $70 \%$, respectively.

Key words: nanosilver, raspberry extract, Cladosporium cladosporioides, Aspergillus niger, biocidal activity

Received: 15 October, 2013; revised: 04 December, 2013; accepted: 04 December, 2013; available on-line: 29 December, 2013

\section{INTRODUCTION}

Nanosilver is one of the most thoroughly investigated nanomaterials and owes its popularity to its biocidal properties (Vaidyanathan et al., 2009). Its antimicrobial activity is associated with the characteristic structure of nanoparticles. They are characterized by a high fraction of surface atoms so that nanoparticles have a greater affinity for interactions with thiol groups, which activity entails the destruction of bacteria (Sadeghi et al., 2010). It exhibits a high antifungal activity, for instance against Candida albicans (Kim et al., 2008; Kim et al., 2009) as well as against some others pathogenic strains (Jo et al., 2009; Kim et al., 2012; Xu et al., 2013). Biological activity is therefore a very important factor, and hence nanosilver is used where asepsis is highly desirable. This applies mostly to biomedical engineering, cosmetology, food packaging industry, textile industry, crops, animal husbandry, etc. (Pulit et al., 2011).

Recently, using nanosilver as a biological agent has become increasingly common (Das et al., 2013; Iravani \& Zolfaghari, 2013; Isaak et al., 2013; Korbekandi et al., 2013; Kumar, 2012; Yasin et al., 2013). The consequence of the above is the desire that the synthesis of nanometric silver should follow the principles of "green chemistry". The development of nanosilver preparation processes which would respect "green chemistry" principles was also the intention of the authors of this paper. It was decided to find a solution that would allow the use of one substance acting as both a stabilizing and reducing agent. The existing state of knowledge allows us to provide a compound composed of similar atomic connections, characterized by the analogical properties of gallic acid which is popular substance having both reducing and stabilizing properties. For example, ellagic acid $(2,3,7,8$-tetrahydroxy-chromeno [5,4,3-cde]chromene-5,10dione) is such a substance. Raspberry fruits are a natural source. Its molecule is a dimer of gallic acid and creates a system of four condensed rings. The use of raspberry extract as a source of ellagic acid offers the possibility of direct silver ion reduction, as it has strong antioxidant effects, similar to many other polyphenols (Pulit et al, 2013). Furthermore, minor amounts of other compounds with antioxidant properties, such as gallic acid, ascorbic acid, and quercetin that also contribute to efficiently carrying out the chemical reduction are also present in raspberry extract. The presence of other organic compounds from the group of polyphenols, tannins and flavonols enables stabilization of the emerging suspensions without the need of adding any other agents that inhibit the growth of metallic silver agglomerates (Hakkinen et al., 2000) This initial premise led us to using raspberry extract, which to a considerable extent meets the criteria of being environmentally friendly.

The problem of asepsis is a very important issue as well. Microbial colonization in painted buildings, for instance, causes aesthetic problems and may lead to degradation and peeling of the paint coating (Bellotti et al., 2013). Much more dangerous effects may be caused by the influence of mould on human health. Spores and mycelial fragments escaping from the ground, together with bacteria and dust, form bioaerosols that are a source of air pollution. They may contain toxic compounds called mycotoxins, which cause allergies. Penicillium spp., Aspergillus spp. and Alternaria spp. are to a significant degree responsible for the occurrence of many allergic symptoms and respiratory diseases diagnosed in people exposed to high levels of mould spores and allergens.

This article presents the possibility of using nanosilver particles, obtained basen on raspberry extract, in order to determine their activity against Cladosporium cladosporoides and Aspergillus niger.

e-mail: jolantapulit@indy.chemia.pk.edu.pl

* Presented at the 5th Central European Congress of Life Sciences "EUROBIOTECH 2013", Kraków, Poland. 


\section{MATERIALS AND METHODS}

Obtaining and characteristics of aqueous raspberry extracts. Dried and ground Rubus fruit was the research material. Organic compounds from raspberry fruit were obtained by extraction in the aqueous phase. In order to accomplish this, a round-bottom flask with a reflux condenser placed in a water bath on a magnetic stirrer was used. The input parameters, which were established in order to carry out the extraction process, were as follows: dried raspberry fruit weight $=4.5 \mathrm{~g}$, extraction process duration $=5 \mathrm{~min}$, process temperature $=30^{\circ} \mathrm{C}$. The reactants contained in the round-bottomed flask (dried raspberries and water) were heated to the desired temperature in a water bath and simultaneously stirred with a magnetic stirrer for a predetermined time. After the extraction process, the supernatant was filtered out. The supernatant was analyzed spectrophotometrically (UV-VIS) in the wavelength range of $\lambda=350-800$ $\mathrm{nm}$. Raspberry extract contains polyphenolic compounds, which are responsible for silver ions reduction and stabilization of nanosilver suspension. Total polyphenol content in the supernatant was determined by the Folin-Ciocalteu method (Blainski et al., 2013).

Preparation of nanosilver suspensions based on raspberry extract. Silver nitrate (V), $\mathrm{AgNO}_{3}, 99.90$ $99.99 \%$ pure, was used as a source of silver ions. Sodium hydroxide, $\mathrm{NaOH}, \geq 98 \%$ BioXtra pure was used to adjust the $\mathrm{pH}$. The method consisted in performing the chemical reduction of silver ions from an aqueous silver nitrate (V) solution with the compounds contained in the aqueous extract of the raspberry fruit in the presence of sodium hydroxide as a $\mathrm{pH}$ controller. The indepen-

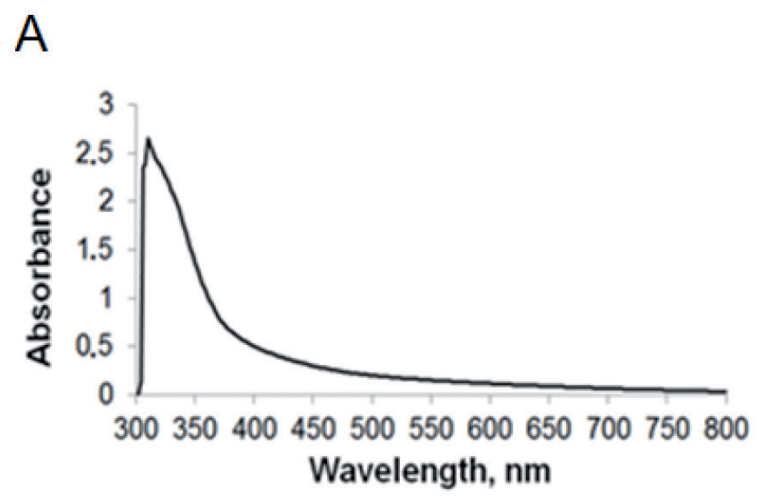

C

a

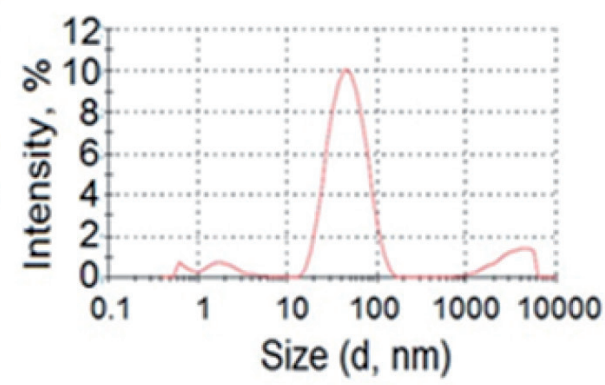

Table 1. Input variables in the process of obtaining nanosilver

\begin{tabular}{ccccc}
\hline System No & $\mathrm{C}_{\mathrm{Ag}}(\mathrm{ppm})$ & $\mathrm{pH}$ & $\begin{array}{l}\text { Stirring } \\
\text { time }(\min )\end{array}$ & $\begin{array}{l}\text { Incubation tem- } \\
\text { perature }\left({ }^{\circ} \mathrm{C}\right)\end{array}$ \\
\hline 1 & 500 & 11 & & 20 \\
\hdashline 2 & 50 & 11 & 5 & 5 \\
\hline 3 & 275 & 7 & & 5 \\
\hline
\end{tabular}

dent variables in the process of obtaining nanosilver are presented in Table 1.

All parameters applied in the process of obtaining silver nanoparticles were established in the preliminary study. In order to differ the results, parameters were varied as well. $1 \mathrm{~cm}^{3}$ of the extract was added to $24 \mathrm{~cm}^{3}$ of room temperature aqueous silver nitrate (V) solution (at the appropriate concentration) in a beaker continuously stirred on a magnetic stirrer. Aqueous sodium hydroxide solution at the concentration of $0.01 \mathrm{M}$ was added in order to achieve the desired $\mathrm{pH}$ of the reaction mixture. The beaker contents were placed in a thermostatic incubator at a predetermined temperature in order to maintain the reduction process in appropriate temperature conditions. The obtained nanometric silver suspensions were analyzed spectrophotometrically. The suspensions were subsequently examined for the purpose of determining the average particle size. The examination was carried out with the use of the dynamic light scattering technique (DLS).

Microbiological examination of the biocidal effect of the obtained nanosilver suspension. Since the antifungal properties of nanosilver obtained with the use of green technology may vary as to their effectiveness,

$\mathrm{B}$

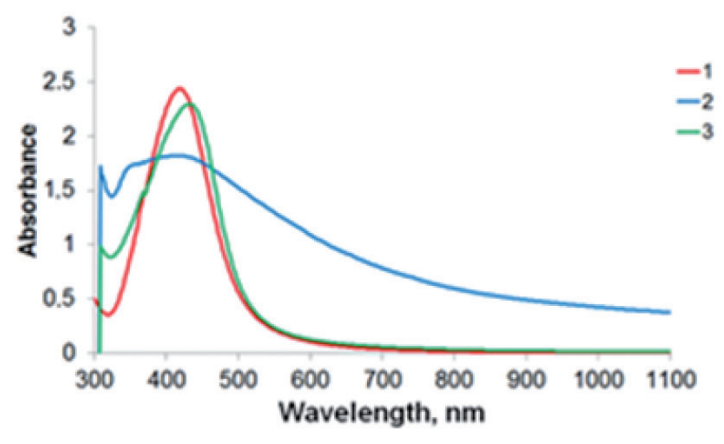

b

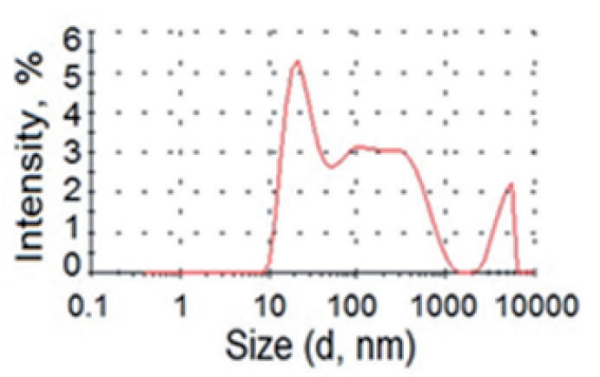

Figure 1. (A) UV-Vis spectra of the obtained raspberry extract; (B) UV-Vis spectra of the obtained nanosilver suspensions (each at the concentration of $50 \mathrm{ppm})$; (C) DLS histograms of the obtained nanosilver suspensions $(\mathrm{a}-$ system $1, \mathrm{~b}-$ system 3$)$. 
the tested preparations were subjected to mycological efficacy resistance analysis in relation to different concentrations of nanostructured silver. Microbiological examination of the biocidal effect of the obtained nanosilver against Cladosporium cladosporoides ATCC 16022 and Aspergillus niger ATCC 16404 was performed. These strains belong to the group of fungi which mostly attack the outdoor paint coatings. The aim was to determine the percentage of the fungal growth reduction. All the relevant tests were carried out in compliance with the Eucast guidelines (Sondi \& Salopek-Sondi, 2004).

Nanosilver suspension was added to the culture media at $40^{\circ} \mathrm{C}$ in the ratio of: $1: 20$ (or other for higher concentration) so that the final volume was $20 \mathrm{ml}$ (agar + suspension). In order to ensure uniform distribution of nanosilver, the agar medium with suspension was remixed in a vortex mixer. Surface inoculating was made by applying $0.1 \mathrm{ml}$ of the appropriate reference strains at a standardized spore concentration of $10^{5}$ spores $/ \mathrm{ml}$ to the Sabouraud agar with different concentrations of nanosilver. The standardization was performed using a microscope (counting spores) and plate method (dilution inoculating). Sabouraud agar with the same amount of spores, but without nanosilver, was used as a control sample. Incubation was carried out at $25^{\circ} \mathrm{C}$ for 10 days. Reduction percentage was determined by a qualitative visual assessment of fungal growth reduction on the test plate surface in set against the control plate.

\section{RESULTS}

Figure 1A presents spectrophotometric data obtained for the received raspberry extract diluted 10 times with distilled water. It is worth noticing that the space between 400 and $500 \mathrm{~nm}$ of wavelength remains empty (without any characteristic peak) so as to leave the possibility of the occurrence of the characteristic nanosilver peak. The obtained extract was investigated by the Folin-Ciocalteu method. The investigation established the content of polyphenols in the extract at $10 \mathrm{mg} / \mathrm{cm}^{3}$.

According to the spectrophotometric data (Fig. 1B), the presence of nanosilver particles in system 2 was not confirmed. It is evidenced by the absence of the characteristic nanosilver peak. That was the reason why only systems 1 and 3 were qualified for further use in the study. On the basis of the data regarding nanoparticles size, it was decided to include only system 1 in the mi-

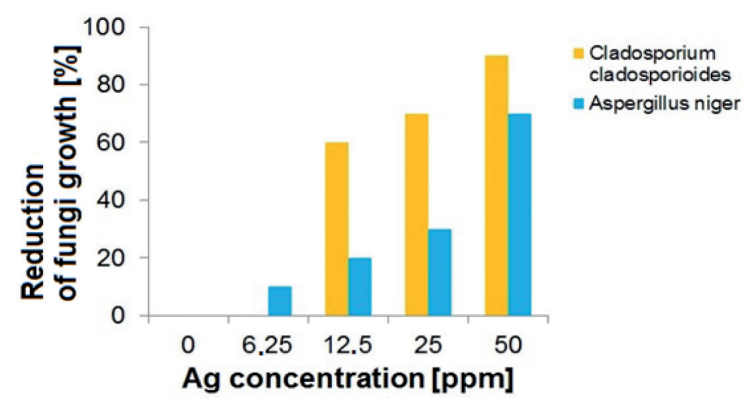

Figure 2. Results of the microbiological tests

crobiological study. The particles in system 3 proved to be too large. (Fig. 1C).

Microbiological results concerning the fungi growth reduction are shown in Figs 2 and 3.

\section{Overview}

Nanosilver suspensions were successfully obtained by using aqueous raspberry extract. Compounds contained in the extract served as reducing agents of silver ions and, in consequence, as the suspension stabilizers. Their amount was determined. The obtained nanosilver suspension was used to inhibit the growth of fungi. The results showed that the obtained suspension was an effective growth inhibition factor against Cladosporium cladosporioides and Aspergillus niger. As demonstrated by the above data, a higher nanosilver concentration induces stronger reduction of fungal growth. Aspergillus niger is a more resistant pathogen of the two examined strains. This strain is known to be one of the most resistant pathogens among fungi. It may result from the activity of chemical components (such as ascorbic acid) which are produced by Aspergillus niger. Nevertheless, the specific resistance mechanism needs to be further investigated in greater detail.

\section{CONCLUSIONS}

A nanosilver suspension was obtained, with particles $60 \mathrm{~nm}$ in size. It was produced with the use of a green method. The assessment of microbiological properties demonstrated the fungicidal activity of nanosilver against

A

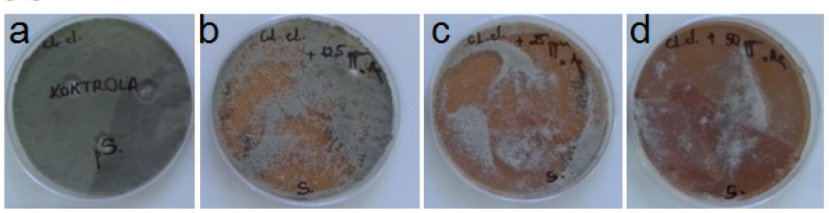

B
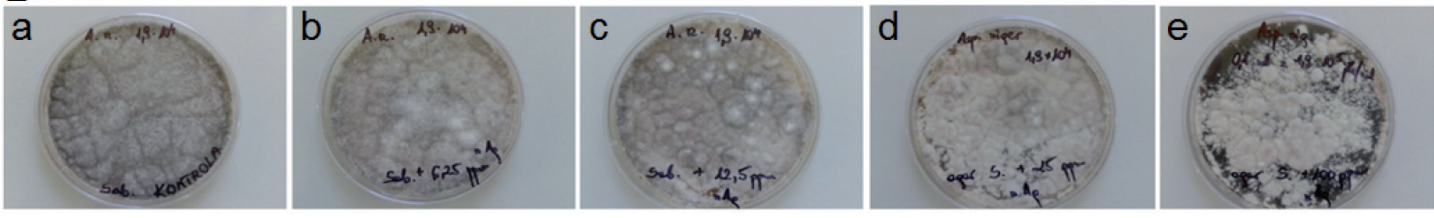

Figure 3. (A) Results of the microbiological tests against Cladosporium cladosporioides using nanosilver suspension at the concentration of: a $-0 \mathrm{ppm}, \mathrm{b}-12.5 \mathrm{ppm}, \mathrm{c}-25 \mathrm{ppm}, \mathrm{d}-50 \mathrm{ppm}$; (B) Results of the microbiological tests against Aspergillus niger using nanosilver suspension at the concentration of: $a-0 \mathrm{ppm}, \mathrm{b}-6.25 \mathrm{ppm}, \mathrm{c}-12.5 \mathrm{ppm}, \mathrm{d}-25 \mathrm{ppm}, \mathrm{e}-50 \mathrm{ppm}$ 
Cladosporium cladosporioides and Aspergillus niger strains. It was found that Aspergillus niger is the more resistant strain of the two. The research results prove that even a low concentration of nanosilver particles makes it possible to achieve a high percentage of growth inhibition. Using nanosilver suspension at the concentration of 50 ppm inhibits the growth of Cladosporium cladosporioides by $90 \%$, and the same concentration causes $70 \%$ growth inhibition of Aspergillus niger. The obtained results confirm the efficiency of using nanosilver as a component of building materials. The use of nanoparticles with antifungal properties in construction materials can bring numerous potential advantages, such as enhancing their hygienic properties, preventing microbial growth and maintaining their mechanical properties.

\section{Acknowledgements}

The work is a part of the project Synthesis and application of innovative nanomaterials with antimicrobial properties supported by the National Centre for Research and Development under the contract no LIDER/03/146/L-3/11/ NCBR/2012 for the period between 2012-2015.

\section{REFERENCES}

Bellotti N, Salvatore L, Deyá C, Del Panno MT, del Amo B, Romagnoli R (2013) The application of bioactive compounds from the food industry to control mold growth in indoor waterborne coatings. Colloids Surf B 104: 140-144.

Blainski A, Lopes GC, de Mello JCP (2013) Application and analysis of the Folin-Ciocalteu method for the determination of the total phenolic content from Limonium Brasiliense 1. Molecules 18: 6852-6865.

Das S, Parida UK, Bindhani BK (2013) Green biosynthesis of silver nanoparticles using Moringa Oleifera leaf. Int I Nanotechnol Appl 3: 51-62.

Hakkinen SH, Karenlampi SO, Mykkanen HM, Heinonen IM, Torronen AR (2000) Ellagic acid content in berries: Influence of domestic processing and storage. Eur Food Res Technol 212: 175-180.
Iravani S, Zolfaghari B (2013) Green synthesis of silver nanoparticles using Pinus eldarica bark extract. BioMed Int Res Article ID 639725, 5 pages, doi:10.1155/2013/639725.

Isaac RSR, Sakthivel G, Murthy C (2013) Green synthesis of gold and silver nanoparticles using Averrhoa bilimbi fruit extract. I Nanotechnol Article ID 906592, 6 pages, doi:10.1155/2013/906592.

Jo YK, Kim BH, Jung G (2009) Antifungal activity of silver ions and nanoparticles on phytopathogenic fungi. Plant Disease 93: 1037-1043.

Kim SW, Jung JH, Lamsal K, Kim YS, Min JS, Lee YS (2012) Antifungal Effects of Silver Nanoparticles (AgNPs) against Various Plant Pathogenic Fungi. Mycobiology 40: 53-58.

Kim KJ, Sung WS, Moon SK, Choi JS, Kim JG, Lee DG (2008) Antifungal effect of silver nanoparticles on dermatophytes. J Microbiol Biotechnol 18: 1482-1484.

Kim KJ, Sung WS, Suh BK, Moon SK, Choi JS, Kim JG, Lee DG (2009) Antifungal activity and mode of action of silver nano-particles on Candida albicans. Biometals 22: 235-242.

Korbekandi H, Asghari G, Jalaver SS, Jalayer MS, Bandegani M (2013) Nanosilver particles production using Juglans Regia (L. Walnut) leaf extract. J Nat Pharm Prod 8: 20-26.

Kumar DA (2012) Rapid and green synthesis of silver nanoparticles using the leaf extracts of Parthenium Hysterophorus: A nover biological approach. Int Res J Pharm 3: 169-173.

Pulit J, Banach M, Kowalski Z (2011) Nanosilver - making difficult decisions. Ecol Chem Eng S 18: 185-196.

Pulit J, Banach M, Zielina M, Laskowska B, Kurleto K (2013) Raspberry extract as both a stabilizer and a reducing agent in environmentally friendly process of receiving colloidal silver. I Nanomater Article ID 563826, 12 pages, doi:10.1155/2013/563826.

Sadeghi B, Jamali M, Kia S, Amininia A, Ghafari S (2010) Synthesis and characterization of silver nanoparticles for antibacterial activity. Int I Nano Dim 1: 119-124.

Sondi I, Salopek-Sondi B (2004) Silver nanoparticles as antimicrobial agent: a case study on E. coli as a model for Gram-negative bacteria. J Colloid Interface Sci 275: 177-182.

Vaidyanathan R, Kalishwaralal K, Gopalram S, Gurunathan S (2009) Nanosilver - the burgeoning therapeutic molecule and its green synthesis. Biotechnol Adv 27: 924-937.

Xu Y, Gao C, Li X, He Y, Zhou L, Pang G, Sun S (2013) In vitro antifungal activity of silver nanoparticles against ocular pathogenic filamentous fungi. J Ocul Pharmacol Ther 29: 270-274.

Yasin S, Liu L, Yao J (2013) Biosynthesis of silver nanoparticles by bamboo leaves extract and their antimicrobial activity. J Fiber Bioeng Informat 6: 77-84. 